\title{
Barrage formation is independent from heterokaryon incompatibility in Verticillium dahliae
}

\author{
Ioannis A. Papaioannou • Milton A. Typas
}

Accepted: 26 August 2014 / Published online: 5 September 2014

(C) Koninklijke Nederlandse Planteziektenkundige Vereniging 2014

\begin{abstract}
Barrage formation has been traditionally used for the assessment of mycelial compatibility in many fungi and has often been assumed to represent a non-self recognition phenotype that is directly associated with vegetative incompatibility in these organisms. In this work, the optimal growth conditions for large-scale studies of barrage formation in the asexual fungus Verticillium dahliae were determined, and they were used for the analysis of a diverse collection comprising 69 isolates of $V$. dahliae and related species. Barrage formation was very frequent on a defined complete agar medium within $V$. dahliae and between species of the genus. However, it was not correlated with the classification of $V$. dahliae isolates into Vegetative Compatibility Groups (VCGs) (based on the standard method using complementary nit mutants), as it was recorded at high frequencies both within and between $V$. dahliae VCGs. The high overall frequency of barrage formation demonstrated the presence of a higher level of mycelial incompatibility in $V$. dahliae than heterokaryon incompatibility assessed by forcing complementary nit mutants to form heterokaryons under selective conditions. The possible association of barrage formation with morphological characteristics of the fungal
\end{abstract}

Electronic supplementary material The online version of this article (doi:10.1007/s10658-014-0525-3) contains supplementary material, which is available to authorized users.

I. A. Papaioannou $\cdot$ M. A. Typas $(\bowtie)$

Department of Genetics and Biotechnology, Faculty of

Biology, National and Kapodistrian University of Athens,

Panepistimiopolis, Athens 15701, Greece

e-mail: matypas@biol.uoa.gr colonies was investigated, and a negative correlation of frequency and intensity of barrages with the isolates' capacity for pigment production was detected.

Keywords Barrage formation · Heterokaryon incompatibility · Mycelial compatibility groups . Mycelial interactions - Non-selective compatibility tests . Vegetative compatibility

\section{Introduction}

Verticillium dahliae Kleb. is a soil-borne phytopathogenic ascomycete that causes vascular wilt in hundreds of plants and billions of dollars in annual crop losses worldwide. The longevity of its microsclerotia in the soil, its vascular habitat, its limited host specialization, and the shortage of resistant host germplasm, have hindered control of the disease. A thorough understanding of the population biology of the fungus is hopefully predicted to facilitate disease management (Pegg and Brady 2002; Klosterman et al. 2009).

Given the asexual nature of $V$. dahliae, the well established parasexual cycle is perceived as the only means of exchange of genetic information between members of this species (Hastie 1964; Puhalla and Mayfield 1974; Typas and Heale 1978). However, even from the first relevant studies, it was evident that when different strains of the fungus come in contact, the formation of viable heterokaryons is frequently prevented by incompatibility systems (Heale 1966; Typas and Heale 1976), resulting in the subdivision of 
the species into compatibility groups. Through the years, a general assumption was made, i.e., that $V$. dahliae isolates belonging to different compatibility groups are genetically isolated and, thus, may represent clonal lineages that possibly differ in physiology and pathogenicity (Leslie 1993; Katan 2000). Different methods have been employed for the characterization of $V$. dahliae populations regarding vegetative compatibility, including: pairing of complementary auxotrophic mutants requiring amino acids, purines/pyrimidines or vitamins (Typas and Heale 1976; Clarkson and Heale 1985); morphological mutants in the pigment biosynthesis pathway (Puhalla 1979; Puhalla and Hummel 1983); and nitrate-nonutilizing (nit) mutants (the compatibility data produced by complementation tests of appropriate mutants of fungal strains are generally referred to as heterokaryon incompatibility results) (Joaquim and Rowe 1990, 1991; Strausbaugh et al. 1992; Katan 2000; Bhat et al. 2003; Jiménez-Díaz et al. 2006; Papaioannou et al. 2013b). Due to the relative ease of selection of spontaneous nit mutants on chlorate-amended growth media, the latter technique has been by far the most widely used method during the last two decades for the classification of $V$. dahliae isolates into five main Vegetative Compatibility Groups (VCGs); of these, VCGs 1, 2, and 4 have been further subdivided depending on the vigour of complementation (Joaquim and Rowe 1990; Bhat et al. 2003). More recently, considerable progress has been made in the elucidation of molecular and phylogenetic relations between VCGs and their subgroups, revealing a previously unanticipated complexity in the relations within VCGs and between subgroups, e.g., VCGs 2 and 4 were shown to consist of molecularly distinct subgroups with complex inter-VCG relationships, and VCG subgroup 2B has been clearly demonstrated to be polyphyletic (Collado-Romero et al. 2006, 2008; Martin 2010; Papaioannou et al. 2013a, b).

Another approach that has been followed for the population-level assessment of vegetative compatibility in many fungi is the analysis of barrage (or demarcation or aversion zone or line) formation, which represents a type of non-self recognition. Based on the study of barrage formation between wild-type colonies, fungal isolates can be assigned to mycelial compatibility groups (Leslie 1993; Smith et al. 2006). This method is based on the general assumption that vegetatively incompatible isolates of a fungal species form a barrage at the interface where confronting hyphae of colonies interact (various types of barrages have been described for different fungi, usually involving a central darkly pigmented area and/or a thicker and higher mycelial layer), whereas compatible pairs are typically predicted to grow into each other without any obvious morphological alteration (Esser and Meinhardt 1984; Worrall 1997). In contrast to inhibition zones that are free of hyphae due to the secretion and sensing of inhibitory molecules, the barrage formation requires hyphal fusion and cytoplasmic mixing between the hyphae of the incompatible confronted colonies, leading to abnormal or even lethal fusions (Esser 2006). Barrage formation has been regarded as conceptually equivalent to heterokaryon incompatibility (Leslie 1993), and the two phenomena have been often considered to be different manifestations of the same vegetative incompatibility function. However, different reports have reached contradictory conclusions regarding the concordance between heterokaryon incompatibility and barrage formation. A high correlation has been observed in Podospora anserina and Fusarium graminearum (Rizet 1952; McCallum et al. 2004), but discrepancies between the two tests have been reported in other cases. In Neurospora crassa, barrage formation is not associated with four tested heterokaryon incompatibility genes (Micali and Smith 2003), and a perfect correlation between the two phenomena is also absent in Sclerotinia sclerotiorum (Ford et al. 1995; Jo et al. 2008). A similar inconsistency between barrage formation and heterokaryon incompatibility was also demonstrated for one of the six known vegetative incompatibility loci of Cryphonectria parasitica, indicating that the two phenomena are not different manifestations of the same process (Smith et al. 2006).

The purposes of the present study were: (i) to test barrage formation in Verticillium species for the first time, under different growth conditions, and to determine the optimal conditions for a large-scale study; (ii) to analyze a diverse collection of isolates of $V$. dahliae and relative species regarding barrage formation; (iii) to compare mycelial compatibility of $V$. dahliae isolates based on barrage formation with their heterokaryon compatibility classification according to the traditional nit mutants-based complementation procedure; and (iv) to test the possibility of an association between mycelial compatibility behaviour and the isolates' capacity for pigmentation and other morphological characteristics. 
Table 1 Verticillium isolates used in this study, with code names, VCGs, original hosts, and geographic origins (sources)

\begin{tabular}{|c|c|c|c|}
\hline Isolate code & $\mathrm{VCG}^{\mathrm{a}}$ & Host & $\begin{array}{l}\text { Origin } \\
\text { (source) }^{\mathrm{b}}\end{array}$ \\
\hline
\end{tabular}

\section{V. dahliae}

T9

V44

V138I

$\operatorname{cotVd} 16$

$\operatorname{cotVd} 47$

V607I (R04)

V661I

(328 v-1)

V666I

(347 v-1)

$\mathrm{PH}$

V320I

egpVd23

ep8

$31 \mathrm{~V}$

115

Ls. 17

V357I (JY)

V16

cotVd18

123-3

Cf. $38^{\mathrm{c}}$

V702I

423-1

$20 \mathrm{~V}$

530-1

$128 \mathrm{~V}$

$25 \mathrm{~V}$ (SS4)

70-21

PCW

BB

131-M

171-4A

V830

Dvd-E6

S39

V304I

$(\cot 120)$

V684I

1A (1)

1A (1)

$$
1 \mathrm{~A}(2)
$$$$
\text { 1A (3) }
$$$$
\text { 1A (3) }
$$

$$
1 \mathrm{~B}(4)
$$

1B (4)

1B (4)

2A (1)

2A (4)

2A (5)

2A (6)

2A (7)

2B (1)

$$
\text { 2B (8) }
$$

2B (4)

2B (9)

cotton

cotton

cotton

cotton

cotton

green ash

cotton

cotton

pistachio

cotton

eggplant

eggplant

melon

cotton

lettuce

cotton

potato

cotton

olive

2B (10)

2B $(11,12)$

chili pepper

2Ba (13)

artichoke

2B, 2A (10)

pistachio

2B, $2 \mathrm{~A}(7)$

tomato

2B, 2A (10)

pepper

2B, 1, 4A (7)

pepper

2AB, 1, 4A

(7)

3 (14)

3 (1)

$4 \mathrm{~A}(1)$

cotton

pepper

pepper

potato

potato

potato

potato

4A (9)

eggplant

4B (1)

soil

4B (2)

4B (13)

cotton

artichoke

$$
\text { USA, CA (A) }
$$

USA, TX (A)

Spain (B)

Turkey (C)

Turkey (C)

USA, MN (B)

Greece (B)

Greece (B)

USA, CA (A)

USA, CA (B)

Turkey (C)

Israel (D)

Greece (E)

Syria (A)

USA, CA (F)

China (B)

Germany (G)

Turkey (C)

Greece (H)

USA, CA (F)

Spain (B)

Greece $(\mathrm{H})$

Greece (E)

Greece (H)

Greece (E)

USA (E)

USA, AZ (A)

USA, CA (A)

USA, ID (A)

USA (B)

USA (B)

USA, OH (G)

Canada (I)

USA, OH (A)

\begin{tabular}{|c|c|c|c|}
\hline Isolate code & $\mathrm{VCG}^{\mathrm{a}}$ & Host & $\begin{array}{l}\text { Origin } \\
\text { (source) }^{b}\end{array}$ \\
\hline 157 & 4B (17) & potato & USA, OR (I) \\
\hline V39 & 4B (9) & sunflower & Germany (G) \\
\hline $1 \mathrm{~V}$ & 4B (7) & cotton & Greece $(\mathrm{E})$ \\
\hline $435-1$ & $4 \mathrm{~B}, 4 \mathrm{~A}(10)$ & tomato & Greece $(\mathrm{H})$ \\
\hline $456-1$ & 4B, 4A (10) & dill & Greece $(\mathrm{H})$ \\
\hline $457-5$ & 4B, 4A (10) & spinach & Greece $(\mathrm{H})$ \\
\hline $\mathrm{Ca} .83$ & $6(11)$ & bell pepper & USA, CA (F) \\
\hline Ca.146 & $6(11)$ & bell pepper & USA, CA (F) \\
\hline Ca.148 & $6(11)$ & bell pepper & USA, CA (F) \\
\hline Cf. $162^{c}$ & $\operatorname{HSI}^{\mathrm{d}}(11,12)$ & chili pepper & USA, CA (F) \\
\hline V13 & HSI (9) & cotton & Spain (G) \\
\hline $\mathrm{Ud} 1-4-1^{\mathrm{e}}$ & - & $\begin{array}{l}\text { Japanese } \\
\text { spikenard }\end{array}$ & Japan (J) \\
\hline TO- $0^{\mathrm{e}}$ & - & tomato & Japan (J) \\
\hline $\mathrm{CA} 26^{\mathrm{e}}$ & - & cabbage & Japan (J) \\
\hline \multicolumn{4}{|l|}{ V. albo-atrum } \\
\hline 220 & - & alfalfa & UK (K) \\
\hline HP & - & potato & Japan (J) \\
\hline Ms.102 & - & alfalfa & USA, PA (F) \\
\hline P10 & - & hop & Germany (L) \\
\hline $\mathrm{T} 2$ & - & hop & Slovenia (L) \\
\hline \multicolumn{4}{|c|}{ V. longisporum } \\
\hline 81 & - & oilseed rape & Denmark $(G)$ \\
\hline CA10 & - & cabbage & Japan (J) \\
\hline Dk-1 & - & $\begin{array}{c}\text { Japanese } \\
\text { radish }\end{array}$ & Japan (J) \\
\hline G19 & - & oilseed rape & Germany (K) \\
\hline $\mathrm{K} 2$ & - & oilseed rape & Russia (M) \\
\hline
\end{tabular}

Israel (B)

Spain (B)
Table 1 (continued)

\section{V. tricorpus}

15

18

19

22

VTR-5 (18) soil

Israel (D)

Israel (D)

Israel (D)

Israel (D)

\section{V. nubilum}

278,734

potato

UK (IMI)

Gibellulopsis nigrescens (formerly $V$. nigrescens)

9

14
18
$\mathrm{VN}-5$ (18) potato

VN-5 (18) Graminae

(potato

field)

VN-10 (18) soil
Israel (D)

Israel (D)

Israel (D) 
Table 1 (continued)

\begin{tabular}{llll}
\hline Isolate code & $\mathrm{VCG}^{\mathrm{a}}$ & Host & $\begin{array}{l}\text { Origin } \\
\text { (source) }^{\mathrm{b}}\end{array}$ \\
\hline 19 & $\mathrm{VN}-11(18)$ & soil & Israel (D) \\
\hline
\end{tabular}

a Data on "bridging" behavior (i.e., complementation to varying degrees with tester strains of more than one VCG subgroups) were available for several $V$. dahliae isolates that were included in this study; these secondary VCG interactions are provided after commas. The reference of studies reporting the VCG assignment of each isolate is provided in brackets, as follows: $1=$ Joaquim and Rowe 1990; 2 = Korolev et al. 2001; 3 = Dervis et al. 2008; $4=$ Collado-Romero et al. 2006; $5=$ Dervis et al. 2009; $6=$ Korolev et al. 2000; 7 = Elena and Paplomatas 1998; $8=$ Papaioannou \& Typas, unpublished data; $9=$ Zeise and von Tiedemann $2001 ; 10=$ Papaioannou et al. 2013b; 11 = Bhat et al. 2003; 12 = Papaioannou et al. 2014; 13 = Jiménez-Díaz et al. 2006; 14 = Joaquim and Rowe $1991 ; 15=$ mentioned by Collado-Romero et al. 2008 as unpublished results; $16=$ Dobinson et al. 1998; $17=$ Dobinson et al. 2000; 18 = Korolev and Katan 1999

b Provided by: A = R. Rowe, OARDC, The Ohio State University, USA; B = M. Jiménez-Gasco, The Pennsylvania State University, USA; C $=$ S. Dervis, University of Mustafa Kemal, Turkey; $\mathrm{D}=\mathrm{T}$. Katan, The Volcani Center, Israel; E = E. Paplomatas, Agriculture University of Athens, Greece; F = K. Subbarao, University of California, Davis, USA; G = A. von Tiedemann, University of Göttingen, Germany; $\mathrm{H}=$ E. Ligoxigakis, Plant Protection Institute, N.AG.RE.F., Greece; I = K. Dobinson, University of Western Ontario, Canada \& Agriculture and Agri-Food, Canada; $\mathrm{J}=\mathrm{T}$. Usami, Chiba University, Japan; K = J. Heale, University of London, UK; L = S. Radišek, Slovenian Institute for Hop Research and Brewing, Slovenia; M = O. Strunnikova, All-Russian Research Institute for Agricultural Microbiology, Russia; IMI = International Mycological Institute, UK (presently Centre for Agricultural Bioscience International, CABI, UK)

${ }^{\mathrm{c}}$ These isolates were initially assigned to VCG 6 by Bhat et al. (2003). The VCG classification of them was later re-examined by Papaioannou et al. (2014), and the revised classification is provided in this Table

${ }^{\mathrm{d}} \mathrm{HSI}=$ heterokaryon self-incompatible

${ }^{\mathrm{e}}$ Reported to bear the MAT1-1-1 mating type idiomorph (Usami et al. 2009)

\section{Materials and methods}

Fungal isolates

Monoconidial cultures from a total of 65 Verticillium isolates $(50 \mathrm{~V}$. dahliae, $5 \mathrm{~V}$. albo-atrum, 5 $V$. longisporum, $4 V$. tricorpus, and $1 V$. nubilum representative strains), as well as 4 isolates of Gibellulopsis nigrescens - formerly $V$. nigrescens - from various hosts and geographic origins were employed in this study (Table 1). Members of all known VCGs of
$V$. dahliae were included in this collection. All isolates were stored as conidial suspensions in Czapek-Dox broth supplemented with $20 \%$ sterile glycerol, at $-80{ }^{\circ} \mathrm{C}$. Actively growing cultures were obtained prior to each experiment by spreading aliquots of the frozen conidial suspensions on PDA plates (Scharlau, Barcelona, Spain) and incubating at $24{ }^{\circ} \mathrm{C}$, in the dark, for 5 days.

Growth media and culture conditions for the assessment of barrage formation

Various combinations of complete (PDA, PCA, CM) and minimal (Czapek-Dox) agar media (described by Typas and Heale 1976), with different light conditions during incubation (a 12-h ambient-light photoperiod or continuous darkness), were tested for the determination of those conditions that favour the production of discrete and reproducible mycelial interactions between confronted hyphae of wild-type isolates. For each pairing, two isolates were point-inoculated (from the edge of 1-week-old colonies grown on CM agar) at a specified distance between inocula on the agar surface, and the plates were incubated at the specified conditions for 10 days. Different distances in the range of $0.5-$ $3.0 \mathrm{~cm}$ between fungal inocula were tried in preliminary tests and assessed for the reproducibility of the results. Initial experiments demonstrated that up to five different isolates could be inoculated at a cruciform pattern and tested on the same plate (Fig. 1), with individual pairings never interfering with each other under these conditions. After the end of the incubation period, barrage formation was recorded, and the intensity of each barrage was estimated in an arbitrary 1-3 ordinal scale (Fig. 1). Class 0 corresponded to compatible interactions, characterized by the gradual intermingling of the confronting colonies with no apparent morphological differentiation. Each pairing was scored in at least two replicates, and self-pairings were included as controls in all experiments.

\section{Morphological characterization and microscopic observations}

Growth rates were determined by measuring the perpendicular diameters of fungal colonies grown on CM agar at daily intervals, and the mean growth rates were calculated for the period of active growth until the end of the second week of incubation. Two weeks after 

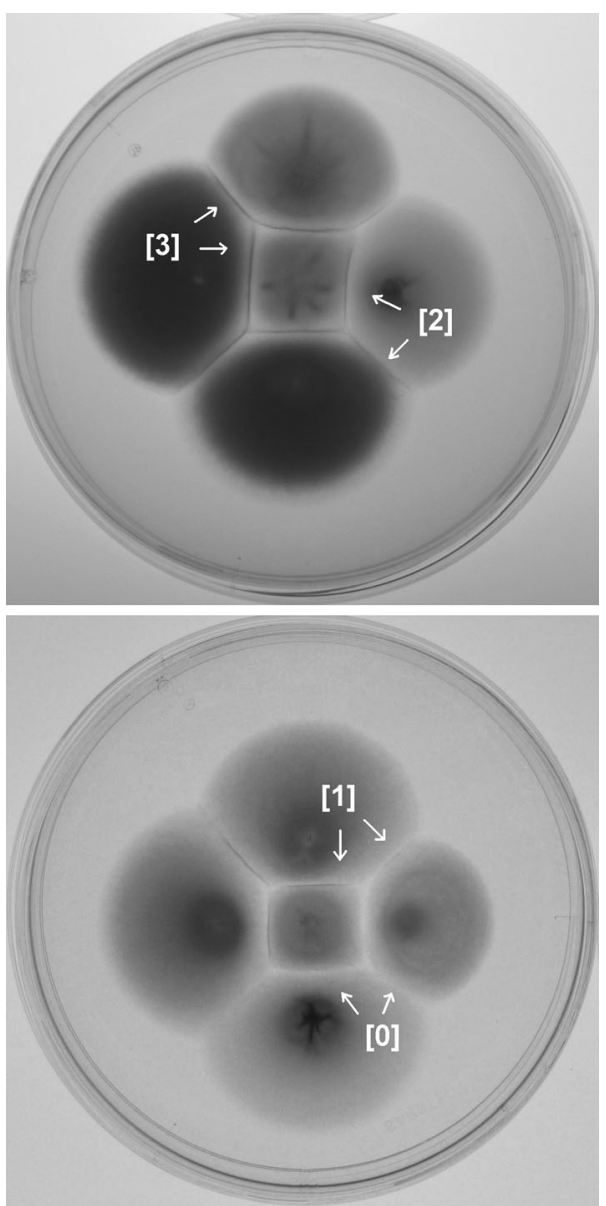

Fig. 1 Examples of barrage formation between colonies of several $V$. dahliae wild-type isolates on CM agar, at the end of the coincubation period (10 days). The three-point (1-3) ordinal graduation (classes provided in square brackets) used for the assessment of the intensity of barrages is illustrated with representative examples (indicated with arrows). Class 0 corresponds to compatible interactions, characterized by the absence of barrages and the gradual intermingling of the compatible colonies with no apparent morphological differentiation; this process had not been completed for the majority of pairings by the end of the 10-day incubation period on CM agar, but was invariably verified with longer incubation for all tested compatible pairings

inoculation on CM agar, colonies were scored in a fourpoint ordinal gradation for hyphal density. A five-point scale was also used to assess pigment production at the bottom of the plates 3 weeks after inoculation. All morphological tests were performed in three replicates per parameter examined for each isolate.

For the live-cell imaging of undisturbed barrages at the microscopic level, representative incompatible pairings of $V$. dahliae isolates were performed on microscopy slides covered with thin layers of solidified
$\mathrm{CM}$ medium, along with compatible pairings which were used as controls. Inoculation and incubation of the slides were performed as described above for the standard barrage formation assays. After appropriate incubation, a drop of sterile water and a coverslip were placed on each slide, above the contact area of the confronting colonies. Alternatively, hyphae within the contact zone were subjected to double staining with the fluorescent dyes propidium iodide and fluorescein diacetate (Sigma-Aldrich Corporation, St. Louis, MO, USA) in order to determine dead or dying cells (Livanos et al. 2012). All samples were examined with a Zeiss Axioplan epifluorescence microscope with appropriate filters, and photos were captured with a Zeiss Axiocam MRc5 digital camera using AxioVs40 V4.40.0 software (Carl Zeiss AG, Oberkochen, Germany). All samples were checked for UV autofluorescence. The same microscope was used with a polarized light source for differential interference contrast (DIC or Nomarski) imaging of stained and non-stained samples.

\section{Statistical analysis}

Statistical tests were conducted with SPSS Statistics 20 software package (IBM Corporation, Armonk, NY, USA). When both normality of data and homogeneity of variances complied with the general assumptions for parametric tests, data were processed with $t$-test or oneway analysis of variance (one-way ANOVA) for dichotomous or multi-group variables, respectively. Post-hoc analysis between groups following ANOVA was accomplished with the Tukey HSD method. The nonparametric Mann-Whitney $U$ test was used for comparisons of datasets not fulfilling any of the two criteria mentioned above.

\section{Results}

Mycelial interactions within and between Verticillium species

During an initial investigation of mycelial reactions between Verticillium wild-type isolates, a collection of 20 isolates of all species were confronted in all possible combinations, on different complete and minimal solid media, and incubated at $24{ }^{\circ} \mathrm{C}$ in the dark or under a 12-h ambient-light photoperiod. The formation of barrages was markedly influenced by both the growth 
medium and the light during incubation (data not shown). Furthermore, the results depended considerably on the exact distance between inocula on the agar surface; the reproducibility of the method among independent repetitions of the pairings was not satisfactory when distances shorter than $1.5 \mathrm{~cm}$ were tested. The most reproducible results, with the most pronounced and discernible barrages, were obtained on CM agar plates, when inocula were placed $1.5 \mathrm{~cm}$ apart and incubated in continuous darkness for 10 days. Thus, these optimal growth conditions were consistently used in all subsequent experiments for the analysis of barrage formation in Verticillium species.

The 69 Verticillium isolates listed in Table 1 were paired in a total of 692 different intra- and inter-isolate pairings (at least two repetitions each; Online Resource 1) under the specific conditions mentioned above, and their mycelial reactions were recorded. Four reaction types were generally observed for all Verticillium species: (i) the formation of a barrage at the contact line of the isolates, i.e., a relatively sharp distinct zone of thick mycelium, occasionally with accumulated dark pigment, of varying degrees of intensity (intensity was assigned in an arbitrary 1-3 scale, with 3 corresponding to the most pronounced barrages; examples are shown in Fig. 1); (ii) mutual intermingling of the colonies with no apparent morphological differentiation (compatible interactions; scored as 0 ); (iii) overgrowth of one colony by the other; and (iv) clear zones devoid of hyphae or consisting of thin mycelium between the confronted colonies. Under these conditions, the presumed barrages between incompatible wild-type colonies were microscopically found to consist of dense bundles of thicklywalled disorganized compartments, at the area where hyphae from the incompatible isolates had come into physical contact and repeatedly fused (Fig. 2). To
Fig. 2 Microscopic

characteristics of hyphae within the contact zones of confronting colonies of $V$. dahliae isolates, in representative compatible (a) and incompatible (b) pairings (isolates 115 and Cf.38 for the compatible reaction; and Ls.17 and V16 for the incompatible one). For each pairing, a DIC micrograph of hyphae is provided on the left, and a photo of the same area of the sample produced with epifluorescence microscopy with propidium iodide staining, is shown on the right. Disorganized, thickly walled, and dead or dying hyphal compartments, which are thus stained with propidium iodide, predominate the contact zone of the incompatible pairing, in contrast to the compatible interaction. Scale bars $=50 \mu \mathrm{m}$

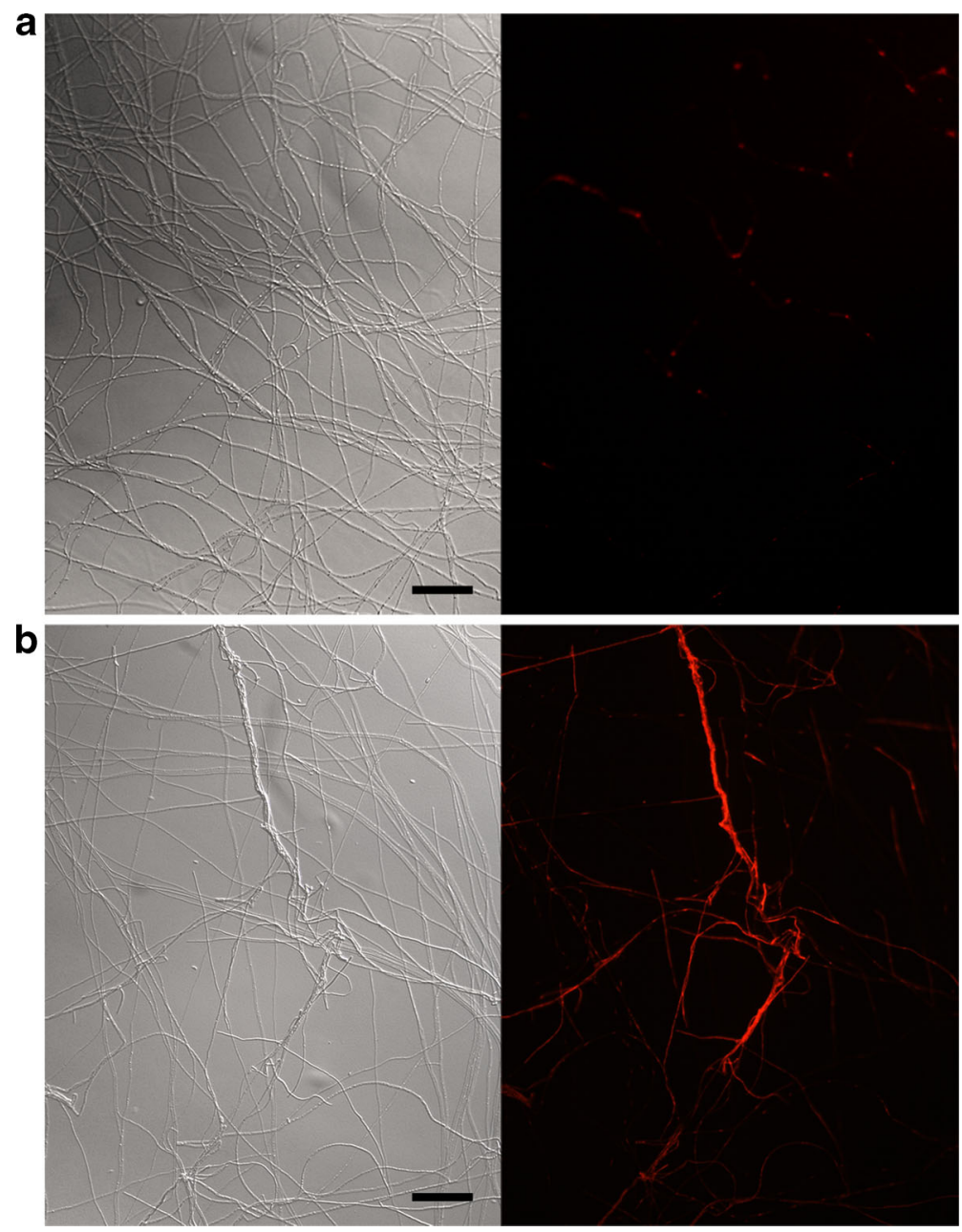


identify dead or dying hyphal compartments, fluorescent staining with propidium iodide - which is not permeant to live cells - was employed and verified with fluorescein diacetate staining, used as a viability probe. The presumed nuclei of only few cells were stained with propidium iodide in all compatible pairings tested as controls, whereas brightly fluorescing dead cells predominated the contact zone of incompatible confronting colonies (Fig. 2). Occasionally, swollen hyphal cells resembling the initial stages of microsclerotia production were also observed along this line of contact, at the end of the incubation period, but mature microsclerotia were never detected. Although the gradual intermingling of compatible colonies was only partially completed for the majority of compatible pairs by the end of the 10-day incubation period on $\mathrm{CM}$ agar (examples are shown in Fig. 1), this introduced no compromise to the accuracy of the assay by visual inspection of plates. To confirm this, compatible pairings were incubated for longer incubation periods and invariably led to completely intermingled colonies, thus validating the accuracy of the initial assay.

The formation of barrages was by far the most frequent reaction type on CM agar (observed in $80.4 \%$ and $75.4 \%$ of pairings within $V$. dahliae or considering all Verticillium species, respectively; Table 2), and, interestingly, it was also encountered in self-pairings with very high frequencies $(90.7 \%$ and $84.8 \%$, respectively). The rest of the reaction types were less frequently observed, with type (iii) being encountered mainly in a few inter-specific pairings. For comparison, 385 pairings of the same strain set (a total of 854 pairings taking repetitions into account) were performed on MM agar (Online Resource 1). A severe reduction in barrage formation was generally observed on MM (14.3\% for all species), with reaction type (iv) being the most prevalent $(41.8 \%)$. Regarding reproducibility of the method that we used for the analysis of barrage formation, the vast majority of pairings yielded identical or very similar results between repetitions. To further validate this observation, 24 randomly selected pairings were repeated in 10 independent trials each; in all cases, the results were absolutely congruent regarding formation of barrages and 80-100\% (averaging 86.3\%) congruent regarding the assessment of barrage intensity (Online Resource 1).

\section{Comparison of barrage formation and VCG classification in $V$. dahliae}

Within $V$. dahliae, barrage formation (i.e., mycelial incompatibility) was compared with heterokaryon incompatibility on the basis of VCG classification using nit mutants. For this, the frequency and intensity of barrages within and among all VCGs of $V$. dahliae, in all possible combinations, were analyzed. Remarkably, no association of barrages with the traditional VCG system was found, with barrages being frequently formed in pairings of isolates belonging to either the same or different VCGs (Table 3). In addition, some isolates were compatible (i.e., they did not form barrages) with only one of a pair of compatible isolates, while others were compatible with both of mutually incompatible strains. These results hampered the classification of the isolates into clear-cut mycelial compatibility groups. Nevertheless, it was found that the frequency and vigour of barrage formation largely depended on the individual isolates tested, with extreme examples being isolates that tended to form barrages with all or most isolates, regardless of their VCG classification (e.g., $V$. dahliae isolates V16, Dvd-E6, and V13; Table 4), and others

Table 2 Frequency (\%) and mean value of intensity (in an arbitrary 1-3 three-point scale; provided in brackets) of barrage formation on CM agar within and between Verticillium species (all individual pairings are listed in Online Resource 1)

\begin{tabular}{|c|c|c|c|c|c|c|}
\hline species & V. dahliae & V. albo-atrum & V.longisporum & V.tricorpus & V.nubilum & G.nigrescens \\
\hline V. dahliae & 80.4 (1.9) & $75.0(1.8)$ & $66.0(1.9)$ & $55.6(1.9)$ & $44.4(1.5)$ & $82.4(1.5)$ \\
\hline V. albo-atrum & & $64.3(2.0)$ & $45.5(1.6)$ & $75.0(1.7)$ & 0 & $100(1.3)$ \\
\hline V. longisporum & & & $64.3(1.5)$ & $62.5(1.5)$ & $33.3(1.5)$ & $25.0(2.0)$ \\
\hline V. tricorpus & & & & 87.5 (1.7) & $100(1.0)$ & $83.3(1.4)$ \\
\hline V. nubilum & & & & & $100(2.5)$ & 0 \\
\hline G. nigrescens & & & & & & $60.0(1.4)$ \\
\hline
\end{tabular}


Table 3 Frequency (\%) and mean value of intensity (in an arbitrary 1-3 three-point scale; provided in brackets) of barrage formation on CM medium within and between VCGs of $V$. dahliae (all individual pairings are listed in Online Resource 1; for clarity, only the primary VCG affinities of "bridging" isolates were used in this analysis)

\begin{tabular}{|c|c|c|c|c|c|c|c|c|c|}
\hline VCG & $1 \mathrm{~A}$ & $1 \mathrm{~B}$ & $2 \mathrm{~A}$ & $2 \mathrm{~B}$ & 3 & $4 \mathrm{~A}$ & $4 \mathrm{~B}$ & 6 & HSI \\
\hline $1 \mathrm{~A}$ & $92.9(2.2)$ & $75.0(1.8)$ & $54.5(2.0)$ & $86.7(1.9)$ & $75.0(1.8)$ & 77.8 (1.9) & $57.1(2.0)$ & $75.0(2.3)$ & $100(2.5)$ \\
\hline $1 \mathrm{~B}$ & & $100(2.3)$ & $33.3(1.0)$ & $66.7(1.3)$ & $100(2.0)$ & $100(1.5)$ & $100(2.5)$ & $100(1.3)$ & 0 \\
\hline $2 \mathrm{~A}$ & & & $92.3(2.4)$ & $72.0(1.8)$ & $75.0(1.6)$ & $100(2.1)$ & $80.0(1.6)$ & 0 & $100(2.8)$ \\
\hline $2 \mathrm{~B}$ & & & & $82.6(2.1)$ & $66.7(1.6)$ & $100(1.7)$ & $71.4(1.8)$ & $100(2.2)$ & $92.3(2.0)$ \\
\hline 3 & & & & & $100(1.9)$ & $100(1.9)$ & $75.0(1.4)$ & $\mathrm{ND}^{\mathrm{a}}$ & $100(2.3)$ \\
\hline $4 \mathrm{~A}$ & & & & & & $78.6(2.2)$ & $84.2(2.1)$ & 0 & $100(2.1)$ \\
\hline 4B & & & & & & & $83.3(1.8)$ & $75.0(1.8)$ & $83.3(2.2)$ \\
\hline 6 & & & & & & & & $100(1.5)$ & $100(1.4)$ \\
\hline $\mathrm{HSI}^{\mathrm{b}}$ & & & & & & & & & $100(2.2)$ \\
\hline
\end{tabular}

${ }^{\mathrm{a}} \mathrm{ND}=$ not determined

${ }^{\mathrm{b}} \mathrm{HSI}=$ heterokaryon self-incompatible

that mostly failed to generate any barrage (e.g., V. dahliae isolates cotVd47 and 131-M, and V. longisporum Dk-1).

Correlation between mycelial compatibility and colony morphology

To test the possibility of a correlation between mycelial compatibility and colony morphology, a set of 26 isolates (including representative strains of all $V$. dahliae VCGs) were analyzed in terms of colony growth rate, hyphal density, and pigmentation on solid CM medium (Table 4). Barrage formation was not related to growth rate and hyphal density, but statistically significant differences were identified in both barrage frequency ( $p=$ $0.001)$ and intensity $(p=0.008)$ when isolates with slight and extended pigmentation ability were compared, with the former being more keen producers of barrages (Online Resource 2). When barrage formation in 328 pairings between these 26 isolates was analyzed in relation to the cumulative pigmentation capacity of each pair (Online Resource 3), three groups of pairs were identified (i.e., pairs with slight, moderate, and high total pigmentation, respectively). All of them were significantly different from each other regarding barrage intensity, with the same negative correlation between barrage formation and pigmentation potential (all differences were statistically significant at the $p=$ 0.01 level; Online Resource 4).

\section{Discussion}

In the present study, barrage formation in Verticillium was analyzed on a large scale for the first time, and it was shown to be overall very frequent $(75.4 \%)$ when tested on a complete agar medium. The high frequency observed in $V$. dahliae is in agreement with reports on other fungi (e.g., $70 \%$ in Botrytis cinerea and up to $91.7 \%$ in Sclerotium rolfsii; Korolev et al. 2008; Remesal and Navas-Cortés 2012). Our results demonstrated that mycelial reactions cannot be used to distinguish Verticillium species, as varying proportions of compatible interactions were recorded in almost all combinations of different species. This may be due to the common ancestry and close genetic relationships between Verticillium species, and it is in agreement with the study of barrage formation in F. graminearum in relation to $F$. pseudograminearum (Akinsanmi et al. 2008). The promotion of barrage formation in Verticillium species on a complete medium that is rich in carbon and nitrogen sources is in contrast to what was observed for N. crassa, where it was shown that a lownitrogen minimal medium is required for efficient barrage formation (Micali and Smith 2003). This indicates that the genes involved in barrage formation in the two fungi are under different regulatory pathways.

No significant correlation of barrage formation with heterokaryon incompatibility was detected for any VCG of $V$. dahliae, with barrages being formed at substantial frequencies both within and among different VCGs. The 
Table 4 Morphological characterization of selected Verticillium isolates and barrage formation frequency $(\%)$ and mean value of intensity (in an arbitrary 1-3 three-point scale; provided in brackets) of all different pairings in this study that involved each of the isolates (isolates of each species are sorted according to ascending pigmentation ability)

${ }^{\mathrm{a}}$ Mean value of growth rate for the period of active growth of the colony on CM agar, until the end of the second week after inoculation

${ }^{\mathrm{b}}$ Assessed 2 weeks after inoculation on $\mathrm{CM}$ agar, in an arbitrary $1-4$ scale

${ }^{\mathrm{c}}$ Assessed 3 weeks after inoculation on CM agar, in an arbitrary 0-4 scale

${ }^{\mathrm{d}}$ Assessed 10 days after inoculation of pairings, in an arbitrary $1-$ 3 scale. For each isolate, the percent frequency of barrage formation is provided, followed by the mean intensity of formed barrages (in brackets), and - after the semicolon - the number of different pairings that involved each isolate and was considered in the calculations

\begin{tabular}{|c|c|c|c|c|}
\hline Isolate code & $\begin{array}{l}\text { Growth rate }^{a} \\
(\mathrm{~cm} / \text { day })\end{array}$ & $\begin{array}{l}\text { Hyphal } \\
\text { density }^{b} \\
(1-4)\end{array}$ & $\begin{array}{l}\text { Pigmentation }^{\mathrm{c}} \\
(0-4)\end{array}$ & $\begin{array}{l}\text { Barrage formation } \\
\text { frequency and } \\
\text { intensity }\end{array}$ \\
\hline \multicolumn{5}{|l|}{ V. dahliae } \\
\hline V320I & 0.4 & 4 & 0 & 88.9 (2.3); 36 \\
\hline $530-1$ & 0.3 & 4 & 1 & 67.9 (1.6); 28 \\
\hline Cf.162 & 0.4 & 4 & 1 & 83.3 (1.8); 30 \\
\hline V44 & 0.3 & 1 & 2 & $82.4(2.0) ; 34$ \\
\hline Ls.17 & 0.5 & 4 & 2 & $93.3(2.0) ; 30$ \\
\hline V16 & 0.4 & 4 & 2 & $100(2.1) ; 31$ \\
\hline $\mathrm{BB}$ & 0.4 & 2 & 2 & $90.3(1.8) ; 31$ \\
\hline Dvd-E6 & 0.5 & 4 & 2 & $97.0(2.1) ; 33$ \\
\hline V830 & 0.5 & 4 & 2 & $91.7(1.8) ; 36$ \\
\hline V13 & 0.5 & 4 & 2 & 95.0 (2.2); 40 \\
\hline T9 & 0.4 & 1 & 3 & $81.8(2.0) ; 33$ \\
\hline $70-21$ & 0.4 & 2 & 3 & 86.5 (1.8); 37 \\
\hline V684I & 0.4 & 3 & 3 & $90.6(1.8) ; 32$ \\
\hline Ca.83 & 0.4 & 2 & 3 & 71.4 (1.4); 7 \\
\hline $\mathrm{PH}$ & 0.4 & 3 & 4 & 60.6 (1.9); 33 \\
\hline V702I & 0.4 & 4 & 4 & $70.4(2.0) ; 27$ \\
\hline $25 \mathrm{~V}(\mathrm{SS} 4)$ & 0.4 & 3 & 4 & 80.0 (1.4); 35 \\
\hline 115 & 0.5 & 2 & 4 & $61.1(1.3) ; 36$ \\
\hline PCW & 0.4 & 2 & 4 & $53.6(1.5) ; 28$ \\
\hline S39 & 0.4 & 3 & 4 & $69.0(1.7) ; 29$ \\
\hline CA26 & 0.4 & 3 & 4 & 62.9 (1.4); 35 \\
\hline TO-0 & 0.3 & 3 & 4 & $40.0(1.3) ; 30$ \\
\hline \multicolumn{5}{|l|}{ V. albo-atrum } \\
\hline $\mathrm{T} 2$ & 0.4 & 3 & 1 & $97.0(2.0) ; 32$ \\
\hline Ms.102 & 0.4 & 2 & 3 & $45.2(1.4) ; 31$ \\
\hline \multicolumn{5}{|l|}{ V. longisporum } \\
\hline $\mathrm{K} 2$ & 0.4 & 2 & 0 & 71.4 (1.7); 35 \\
\hline 81 & 0.4 & 4 & 3 & $75.8(2.0) ; 33$ \\
\hline
\end{tabular}

frequent barrage formation even within VCGs indicates that mycelial compatibility is more restricted in $V$. dahliae populations than heterokaryon compatibility. The apparent lack of association implies that the two processes reveal genetic diversity of $V$. dahliae populations at different levels, and the diversity of the results (e.g., isolates that did not form barrages with anyone of mutually "incompatible" isolates, the presence of "super-compatible" and generally "incompatible" isolates) demonstrates that mycelial interaction is probably governed by multiple and putatively quantitative genetic factors. Similar data have also been reported for other fungi, e.g., S. sclerotiorum, S. homoeocarpa, N. crassa,
C. parasitica and B. cinerea (Ford et al. 1995; Micali and Smith 2003; Smith et al. 2006; Jo et al. 2008; Korolev et al. 2008), suggesting altogether that the traditionally presumed analogy between mycelial and heterokaryon incompatibility is not a general rule for ascomycetes.

An interesting finding in this work is that barrage formation in our experiments was frequent even in selfpairings of the tested Verticillium isolates, similarly with previous observations on other fungi, e.g., F. oxysporum, F. graminearum, S. sclerotiorum, and Rosellinia necatrix (Page 1961; Tariq et al. 1985; McCallum et al. 2004; Ikeda et al. 2011). Given that 
the results we have obtained for the study of barrage formation in this work were overall highly reproducible, it is considered unlikely that the dark lines observed in self-pairings were due to random mycelial interactions rather than to genetically controlled recognition mechanisms. Similarly, the alternative hypothesis that these lines might merely correspond to the development of resting structures between the confronting colonies due to the local exhaustion of nutrients is also unlikely, since it was shown in our work that dead or dying hyphal compartments resulting from multiple lethal fusions predominated the contact area of incompatible pairings, and mature microsclerotia were never observed. Although the genetic basis of this phenomenon is still elusive, it could be hypothesized that the barrages observed in self-pairings are related to the phenomenon of partial or late heterokaryon compatibility that is frequently encountered in $V$. dahliae populations, even between complementary mutants of the same strain (Typas 1983; Joaquim and Rowe 1990, 1991; Daayf et al. 1995; Papaioannou et al. 2013b). The notion of incomplete compatibility in Verticillium is further supported by the recovery of heterokaryon selfincompatible isolates at considerable frequencies (Correll et al. 1988; Collado-Romero et al. 2006; Rataj-Guranowska 2006) and the previous finding that similar strains of other fungi have reduced but not absolutely abolished capacity to form hyphal anastomoses and viable heterokaryons under forced conditions (Correll et al. 1989; Hawthorne and Rees-George 1996).

Based on the observation of immature microsclerotia occasionally developing along the contact line of confronted colonies, we tested the hypothesis that an association might exist between barrage formation and main morphological characteristics of the isolates, mainly the capacity for pigmentation, which is known to be restricted to microsclerotia in $V$. dahliae (Pegg and Brady 2002). A negative correlation between the isolates' ability to accumulate dark pigments and the frequency and intensity of barrage formation was indeed detected, although most isolates require longer periods of incubation to fully express their pigmentation potential in their colonies' phenotype than the incubation period of 10 days used in our assay. It is presently not known whether this association would be significant in more extended collections of Verticillium isolates. However, it might be related to evidence from other fungi with coloured or dark mycelium in culture, such as members of the Botryosphaeriaceae and
Cryphonectriaceae, which tend to produce more pronounced barrages than fungi with light coloured mycelium (Burgess et al. 2009). These examples and the situation described in the present study for Verticillium indicate opposite correlations between barrage formation and pigmentation in different groups of fungi (i.e., a negative correlation for Verticillium and a positive one for Botryosphaeriaceae and Cryphonectriaceae members). Overall, these observations together suggest that barrage formation in fungi may be positively or negatively related to pigmentation ability and may thus indicate the presence of an unexplored putative link between mycelial compatibility behavior and morphogenesis of the fungal colony, which welcomes future study.

The extended incongruence that was demonstrated between barrage formation and heterokaryon incompatibility in $V$. dahliae suggests that the two phenomena are controlled by different-although possibly overlapping - genetic systems. Discrepancies between compatibility data for $V$. dahliae populations that were produced with the application of different methods have also been reported in the past. For example, a lower level of compatibility was revealed with the use of morphological mutants of $V$. dahliae isolates in non-forced tests than with the use of amino acid-, purine-/pyrimidine- or vitamin-requiring auxotrophic mutants in forced complementation trials, which generally demonstrated the existence of compatibility at a more pronounced level in populations of the fungus (Typas and Heale 1976; Puhalla 1979; Puhalla and Hummel 1983; Clarkson and Heale 1985). Even incompatible pairs of Verticillium isolates were successfully forced to "artificial" heterokaryon and diploid formation with the techniques of protoplast fusion and microinjection, thus denoting that at least part of the incompatibility barriers in Verticillium species are cell wall-related (Typas 1983). It could be speculated that the discrepancies observed between different methods for the assessment of vegetative incompatibility can be attributed - at least in part - to the fundamentally different criteria that are involved in the assessment of compatibility, i.e., formation of heterokaryons under different levels of selective pressure. Hastie and Heale (1984) have postulated that incompatibility barriers might probably be overcome to some extent under conditions of different selection pressure to permit hyphal fusions and the establishment of heterokaryons only under certain, forced conditions. If future research further substantiates this hypothesis, then both our 
understanding of the population biology of $V$. dahliae and other fungi, and our methodological toolbox to unravel the compatibility behavior of these organisms, are predicted to advance significantly.

Acknowledgments The authors would like to thank all individuals listed in the footnote of Table 1 who provided fungal isolates that were used in this study. Furthermore, Prof. P. Apostolakos and Dr. P. Livanos (Faculty of Biology, National and Kapodistrian University of Athens) are gratefully acknowledged for their valuable help with live-cell imaging and fluorescent staining techniques. I. A. Papaioannou and this research have been cofinanced by the European Union (European Social Fund - ESF) and Greek national funds through the Operational Program "Education and Lifelong Learning" of the National Strategic Reference Framework (NSRF) - Research Funding Program: Heracleitus II. Investing in knowledge society through the European Social Fund.

\section{References}

Akinsanmi, O. A., Backhouse, D., Simpfendorfer, S., \& Chakraborty, S. (2008). Mycelial compatibility reactions of Australian Fusarium graminearum and F. pseudograminearum isolates compared with AFLP groupings. Plant Pathology, 57, 251-261.

Bhat, R. G., Smith, R. F., Koike, S. T., Wu, B. M., \& Subbarao, K. V. (2003). Characterization of Verticillium dahliae isolates and wilt epidemics of pepper. Plant Diseases, 87, 789-797.

Burgess, T., Bihon, W., Wingfield, M. J., \& Wingfield, B. D. (2009). A simple and rapid method to determine vegetative compatibility groups in fungi. Inoculum, 60, 1-2.

Clarkson, J. M., \& Heale, J. B. (1985). Heterokaryon compatibility and genetic recombination within a host plant between hop isolates of Verticillium albo-atrum. Plant Pathology, 34, $129-138$

Collado-Romero, M., Mercado-Blanco, J., Olivares-García, C., Valverde-Corredor, A., \& Jiménez-Díaz, R. M. (2006). Molecular variability within and among Verticillium dahliae vegetative compatibility groups determined by fluorescent amplified fragment length polymorphism and polymerase chain reaction markers. Phytopathology, 96, 485-495.

Collado-Romero, M., Mercado-Blanco, J., Olivares-García, C., \& Jiménez-Díaz, R. M. (2008). Phylogenetic analysis of Verticillium dahliae vegetative compatibility groups. Phytopathology, 98, 1019-1028.

Correll, J. C., Gordon, T. R., \& McCain, A. H. (1988). Vegetative compatibility and pathogenicity of Verticillium albo-atrum. Phytopathology, 78, 1017-1021.

Correll, J. C., Klittich, C. J. R., \& Leslie, J. F. (1989). Heterokaryon self-incompatibility in Gibberella fujikoroi (Fusarium moniliforme). Mycological Research, 93, 21-27.

Daayf, F., Nicole, M., \& Geiger, J.-P. (1995). Differentiation of Verticillium dahliae populations on the basis of vegetative compatibility and pathogenicity on cotton. European Journal of Plant Pathology, 101, 69-79.
Dervis, S., Kurt, S., Soylu, S., Erten, L., Soylu, E. M., Yıldız, M., \& Tok, F. M. (2008). Vegetative compatibility groups of Verticillium dahliae from cotton in the southeastern Anatolia region of Turkey. Phytoparasitica, 36, 74-83.

Dervis, S., Yetisir, H., Yıldırım, H., Tok, F. M., Kurt, S., \& Karaca, F. (2009). Genetic and pathogenic characterization of Verticillium dahliae isolates from eggplant in Turkey. Phytoparasitica, 37, 467-476.

Dobinson, K. F., Patterson, N. A., White, G. J., \& Grant, S. (1998). DNA fingerprinting and vegetative compatibility analysis indicate multiple origins for Verticillium dahliae race 2 tomato isolates from Ontario, Canada. Mycological Research, 102, 1089-1095.

Dobinson, K. F., Harrington, M. A., Omer, M., \& Rowe, R. C. (2000). Molecular characterization of vegetative compatibility group $4 \mathrm{~A}$ and $4 \mathrm{~B}$ isolates of Verticillium dahliae associated with potato early dying. Plant Diseases, 84, 1241-1245.

Elena, K., \& Paplomatas, E. J. (1998). Vegetative compatibility groups within Verticillium dahliae isolates from different hosts in Greece. Plant Pathology, 47, 635-640.

Esser, K. (2006). Heterogenic incompatibility in fungi. In U. Kües \& R. Fischer (Eds.), The Mycota I. Growth, differentiation and sexuality (2nd ed., pp. 141-165). Berlin: Springer.

Esser, K., \& Meinhardt, F. (1984). Barrage formation in fungi. In H. F. Linskens \& J. Heslop-Harrison (Eds.), Cellular interactions. Encyclopedia of plant physiology (pp. 350-361). Berlin: Springer.

Ford, E. J., Miller, R. V., \& Sherwood, J. E. (1995). Heterokaryon formation and vegetative compatibility in Sclerotinia sclerotiorum. Mycological Research, 99, 241-247.

Hastie, A. C. (1964). The parasexual cycle in Verticillium alboatrum. Genetical Research, 5, 305-315.

Hastie, A. C., \& Heale, J. B. (1984). Genetics of Verticillium. Phytopathologia Mediterranea, 23, 130-162.

Hawthorne, B. T., \& Rees-George, J. (1996). Use of nitrate nonutilizing mutants to study vegetative incompatibility in Fusarium solani (Nectria haematococca), especially members of mating populations I, V and VI. Mycological Research, 100, 1075-1081.

Heale, J. B. (1966). Heterokaryon synthesis and morphogenesis in Verticillium. Journal of General Microbiology, 45, 419-427.

Ikeda, K., Inoue, K., Nakamura, H., Hamanaka, T., Ohta, T., Kitazawa, H., Kida, C., Kanematsu, S., \& Park, P. (2011). Genetic analysis of barrage line formation during mycelial incompatibility in Rosellinia necatrix. Fungal Biology, 115, $80-86$.

Jiménez-Díaz, R. M., Mercado-Blanco, J., Olivares-García, C., Collado-Romero, M., Bejarano-Alcázar, J., Rodríguez-Jurado, D., et al. (2006). Genetic and virulence diversity in Verticillium dahliae populations infecting artichoke ineastern-central Spain. Phytopathology, 96, 288-298.

Jo, Y.-K., Chang, S. W., Rees, J., \& Jung, G. (2008). Reassessment of vegetative compatibility of Sclerotinia homoeocarpa using nitrate-nonutilizing mutants. Phytopathology, 98, 108-114.

Joaquim, T. R., \& Rowe, R. C. (1990). Reassessment of vegetative compatibility relationships among strains of Verticillium dahliae using nitrate-nonutilizing mutants. Phytopathology, 80, 1160-1166. 
Joaquim, T. R., \& Rowe, R. C. (1991). Vegetative compatibility and virulence of strains of Verticillium dahliae from soil and potato plants. Phytopathology, 81, 552-558.

Katan, T. (2000). Vegetative compatibility in populations of Verticillium - an overview. In E. C. Tjamos, R. C. Rowe, J. B. Heale, \& D. R. Fravel (Eds.), Advances in Verticillium: research and disease management (pp. 69-86). St. Paul: American Phytopathological Society Press.

Klosterman, S. J., Atallah, Z. K., Vallad, G. E., \& Subbarao, K. V. (2009). Diversity, pathogenicity and management of Verticillium species. Annual Review of Phytopathology, 47, 39-62.

Korolev, N., \& Katan, T. (1999). Vegetative compatibility grouping in Verticillium nigrescens and V. tricorpus. Mycological Research, 103, 65-76.

Korolev, N., Katan, J., \& Katan, T. (2000). Vegetative compatibility groups of Verticillium dahliae in Israel: their distribution and association with pathogenicity. Phytopathology, 90, 529566.

Korolev, N., Pérez-Artés, E., Bejarano-Alcázar, J., RodríguezJurado, D., Katan, J., Katan, T., \& Jiménez-Díaz, R. M. (2001). Comparative study of genetic diversity and pathogenicity among populations of Verticillium dahliae from cotton in Spain and Israel. European Journal of Plant Pathology, 107, 443-456.

Korolev, N., Elad, Y., \& Katan, T. (2008). Vegetative compatibility grouping in Botrytis cinerea using sulphate non-utilizing mutants. European Journal of Plant Pathology, 122, 369383.

Leslie, J. F. (1993). Fungal vegetative compatibility. Annual Review of Phytopathology, 31, 127-150.

Livanos, P., Galatis, B., Quader, H., \& Apostolakos, P. (2012). Disturbance of reactive oxygen species homeostasis induces atypical tubulin polymer formation and affects mitosis in root-tip cells of Triticum turgidum and Arabidopsis thaliana. Cytoskeleton, 69, 1-21.

Martin, F. N. (2010). Mitochondrial haplotype analysis as a tool for differentiating isolates of Verticillium dahliae. Phytopathology, 100, 1231-1239.

McCallum, B. D., Tekauz, A., \& Gilbert, J. (2004). Barrage zone formation between vegetatively incompatible Fusarium graminearum (Gibberella zeae) isolates. Phytopathology, 94, 432-437.

Micali, C. O., \& Smith, M. L. (2003). On the independence of barrage formation and heterokaryon incompatibility in Neurospora crassa. Fungal Genetics and Biology, 38, 209219.

Page, O. T. (1961). Variation in the banana-wilt pathogen, Fusarium oxysporum f. cubense. Canadian Journal of Botany, 39, 545-557.

Papaioannou, I. A., Dimopoulou, C. D., \& Typas, M. A. (2013a). Structural and phylogenetic analysis of the rDNA intergenic spacer region of Verticillium dahliae. FEMS Microbiology Letters, 347, 23-32.

Papaioannou, I. A., Ligoxigakis, E. K., Vakalounakis, D. J., Markakis, E. A., \& Typas, M. A. (2013b). Phytopathogenic, morphological, genetic and molecular characterization of a Verticillium dahliae population from
Crete, Greece. European Journal of Plant Pathology, 136, 577-596.

Papaioannou, I. A., Dimopoulou, C. D., \& Typas, M. A. (2014). "Cryptic" group-I introns in the nuclear SSU-rRNA gene of Verticillium dahliae. Current Genetics, 60, 135-148.

Pegg, G. F., \& Brady, B. L. (2002). Verticillium wilts. New York: CABI Publishing.

Puhalla, J. E. (1979). Classification of isolates of Verticillium dahliae based on heterokaryon incompatibility. Phytopathology, 69, 1186-1189.

Puhalla, J. E., \& Hummel, M. (1983). Vegetative compatibility groups within Verticillium dahliae. Phytopathology, 73, 1305-1308.

Puhalla, J. E., \& Mayfield, J. E. (1974). The mechanisms of heterokaryotic growth in Verticillium dahliae. Genetics, 76, 411-422.

Rataj-Guranowska, M. (2006). Vegetative compatibility in Verticillium dahliae from several European countries. Phytopathologia Polonica, 42, 5-12.

Remesal, E., \& Navas-Cortés, J. A. (2012). An improved growth medium to assess mycelial compatibility groups in Sclerotium rolfsii. Journal of Phytopathology, 160, 237-242.

Rizet, G. (1952). Les phénomènes de barrage chez Podospora anserina. I. Analyse génétique des barrages entre les souches $S$ et $s$. Review of Cytology Bioliology Végétale, 13, 51-92.

Smith, M. L., Gibbs, C. C., \& Milgroom, M. G. (2006). Heterokaryon incompatibility function of barrage-associated vegetative incompatibility genes (vic) in Cryphonectria parasitica. Mycologia, 98, 43-50.

Strausbaugh, C. A., Schroth, M. N., Weinhold, A. R., \& Hancock, J. G. (1992). Assessment of vegetative compatibility of Verticillium dahliae tester strains and isolates from California potatoes. Phytopathology, 82, 61-68.

Tariq, V.-N., Gutteridge, C. S., \& Jeffries, P. (1985). Comparative studies of cultural and biochemical characteristics used for distinguishing species within Sclerotinia. Transactions of the British Mycological Society, 84, 381-397.

Typas, M. A. (1983). Heterokaryon incompatibility and interspecific hybridization between Verticillium alboatrum and Verticillium dahliae following protoplast fusion and microinjection. Journal of General Microbiology, 129, 3043-3056.

Typas, M. A., \& Heale, J. B. (1976). Heterokaryosis and the role of cytoplasmic inheritance in dark resting structure formation in Verticillium spp. Molecular General Genetics, 146, 17-26.

Typas, M. A., \& Heale, J. B. (1978). Heterozygous diploid analyses via the parasexual cycle and a cytoplasmic pattern of inheritance in Verticillium species. Genetical Research, 31, 131-144.

Usami, T., Itoh, M., \& Amemiya, Y. (2009). Asexual fungus Verticillium dahliae is potentially heterothallic. Journal of General Plant Pathology, 75, 422-427.

Worrall, J. J. (1997). Somatic incompatibility in basidiomycetes. Mycologia, 89, 24-36.

Zeise, K., \& von Tiedemann, A. (2001). Morphological and physiological differentiation among vegetative compatibility groups of Verticillium dahliae in relation to $V$. longisporum. Journal of Phytopathology, 149, 469-475. 